\title{
Assessment of the Relationship of $\mathrm{Cd}_{4}$ Count Levels with Lipid Profile and Atherogenic Index of Plasma among Newly Diagnosed HIV Patients in a Tertiary Hospital in Nigeria
}

\author{
Henry Ohem Okpa ${ }^{1,2}$, Efosa Oviasu ${ }^{3,4}$ and Louis Ojogwu $u^{3,4}$. \\ ${ }^{l}$ Department of Internal Medicine, College of Medical Sciences, University of Calabar, Nigeria \\ ${ }^{2}$ Department of Internal Medicine, University of Calabar Teaching Hospital, Calabar, Nigeria \\ ${ }^{3}$ Department of Internal Medicine, College of Medical Sciences, University of Benin, Nigeria \\ ${ }^{4}$ Department of Internal Medicine, University of Benin Teaching Hospital, Benin City, Nigeria
}

\begin{abstract}
Lipid abnormalities have been recorded in both highly active antiretroviral therapy (HAART)-naïve HIV patients and those on HAART, and the presence of this dyslipidemia may lead to increased risk of cardiovascular disease (CVD). The atherogenic index of plasma (AIP) calculated as log of Tgl/HDL is currently being used as a screening tool for dyslipidaemia and is a strong predictor of CVD events. To determine the lipid profile pattern and atherogenic index of plasma among HAART-naïve HIV patients and relationship with $C D_{4}$ count levels. This was a prospective study of

HAART-naïve HIV patients attending the HIV clinic of the University of Benin Teaching Hospital (UBTH), Benin over a 4 month period from March to July 2011. Data generated was analysed using SPSS version 18. A total of 300 patients with significant higher mean age in males than females were recruited for the study (42.4 \pm 10.8 versus $35.8 \pm 8.4, p<0.05)$. HIV- patients with $C_{4}$ cells $<200 / \mu l$ have significant lower age, BMI, and higher AIP with high risk category (AIP > 0.24). HDL is positively correlated with $C D_{4}$ cells, while $T G$ and AIP are negatively correlated ( $p<0.05$ ). In conclusion, HIV-patients with $\mathrm{CD}_{4}$ cells $<200 / \mu l$ (lower immunity) have more lipid abnormalities and high risk AIP category.
\end{abstract}

Keywords: Atherogenic index of plasma, Highly active antiretroviral therapy, HIV, Lipid profile.

\section{Introduction}

Human immunodeficiency Virus (HIV) infection is pandemic worldwide [1]. HIV infection is a worldwide health problem that affects about 34 million men and women. Nigeria, the tenth most populous country in the world and the most populous country in sub-Saharan Africa, has the second highest population of people (about 3 million) living with HIV, after South Africa with about 5.6 million [2].

In the 2015 HIV/Acquired immunodeficiency syndrome (AIDS) estimates in Nigeria, the number of people living with HIV was put at 3.5 million while the prevalence rate of HIV in adults aged $15-49$ years is $3.1 \%$ [3]. AIDS is the leading cause of death in Africa and the fourth leading cause of death worldwide [4]. However, death due to AIDS in Nigeria is 180,000 while 250,000 new HIV infections are recorded, with about $24 \%$ of adults on antiretroviral treatment [3].

The availability of highly active antiretroviral therapy (HAART) has resulted in improved survival of HIV patients. Morbidity and mortality in these patients are therefore expected to come from organic diseases [5]. Metabolic abnormalities are often common among these HIV patients, and include dyslipidemia, diabetes, insulin resistance, altered fat distribution and endothelial dysfunction [6].

Various lipid abnormalities have been recorded in both highly active antiretroviral therapy (HAART)-naïve HIV patients and those on HAART. Hypertriglyceridemia, low total cholesterol (TC), low high density lipoprotein (HDL-c) and reduced low density lipoprotein (LDL-c) levels have been observed in HAART-naïve HIV patients [7] while HIV patients on HAART such as protease inhibitors and nevirapine, the lipid profile pattern showed elevated total cholesterol, triglyceride (TG), low density lipoprotein (LDL-c) and a normal or reduced HDL-c levels [8,9]. Low levels of high density lipoprotein (HDL) were also found to be common especially in those with low CD4 count [10].

In HIV infection, there is an increase in the activity of the cholesterol ester transfer protein which acts to transfer cholesterol from HDL to apo-B - containing proteins, hence resulting in a decrease in HDL [11].

The ratio of triglycerides to HDL-cholesterol (Tgl/HDL-c) is said to correlate inversely with the level of small, dense LDL particles, and atherogenic index of plasma (AIP) calculated as $\log$ of $\mathrm{Tgl} / \mathrm{HDL}$ is a strong predictor of CVD events [12].

A study in Nigeria showed that abnormal AIP is frequent in HAART-naïve HIV patients and is inversely related to their level of immunity and $\mathrm{CD}_{4}$ count was one of the predictor of AIP [13]. 
Hence, the present study aimed to evaluate the lipid profile pattern and atherogenic index of plasma among HAART-naïve HIV patients and relationship with $\mathrm{CD}_{4}$ count levels.

\section{Materials And Methods}

\subsection{Ethical clearance}

Approval for the study protocol was obtained from the ethics committee of the University of Benin Teaching Hospital, Benin City. Only patients who gave informed written consent were recruited.

\subsection{Study setting}

This was a cross - sectional study carried out in the adult HIV clinic and the medical wards of the University Of Benin Teaching Hospital (UBTH), Benin City. The UBTH is one of the major HIV treatment center in Nigeria that is largely supported by the United States presidential emergency fund for AIDS relief (PEPFAR) and receives referrals mainly from states in South - West and South - South Nigeria.

The study was conducted between March and July, 2011. Consecutive newly diagnosed HIV patients who were being enrolled into the HIV treatment program and admitted into the medical wards were recruited after meeting the study criteria and obtaining informed written consent. Patients who were on lipid lowering drugs, known hypertensive and diabetic patients and those with evidence of kidney disease were excluded. Significant history of alcohol use, cigarette smoking and exposure to ART were also part of the exclusion criteria.

Demographic parameters were obtained from the study participants using a structured pretested interviewer administered questionnaire. A history of alcohol use and smoking was also obtained. The weight and height of the patients were measured using a stadiometer with the participants wearing light clothing and without shoes. The body mass index (BMI) was then calculated using the formula: Weight (kg)/height (m2).

The blood pressure was measured with an Accoson sphygmomanometer and a standard sized cuff using the usual methods with the patient sitting quietly. The mean of two readings taken at least 5 minutes apart was recorded as the blood pressure.

\subsection{Definition of variables}

Dyslipidaemia is defined using the World Health Organization criteria as shown below [14]:

- Total cholesterol (TC) $>5.2 \mathrm{mmol} / 1$

- High density lipoprotein (HDL) cholesterol $<1.0 \mathrm{mmol} / 1$ in females and $<0.9 \mathrm{mmol} / 1 \mathrm{in}$ males

- Low density lipoprotein (LDL) cholesterol $>3.5 \mathrm{mmol} / \mathrm{l}$

- Triglyceride $(\mathrm{TG})>1.7 \mathrm{mmol} / 1$

Also, the atherogenic index of plasma was classified as follows [15]:

- Low risk $-0.3-0.1$

- Medium risk $0.1-0.24$

- High risk $>0.24$

\subsection{Investigations}

The HIV status of the patients was confirmed by Western blot and the CD4 cell count was measured using flow cytometry (Partec, Germany).

The blood for serum lipid profile was collected in plain bottles. Serum total cholesterol (TC) and triglyceride (TG) were determined by enzymatic estimation while high density lipoprotein cholesterol 1 (HDL-c) was determined by enzymatic estimation after precipitation. Low density lipoprotein cholesterol (LDL-c) was determined from the values of the aforementioned using the Friedewald's formula [16].

$\mathrm{LDL}=\mathrm{TCx} \mathrm{HDL}(\mathrm{TG} / 5)$

\subsection{Statistical analysis}

Data collected was entered and analyzed using the Statistical Package for Social Sciences (SPSS) version 18.0 (Chicago, USA). Continuous variables were presented as means and standard deviation while categorical variables were recorded as frequencies and percentages. Chi-square test was used to test for the difference between categorical variables while Student's $t$ - test was used to compare means between two groups. One way ANOVA, Pearson's correlation coefficient and multiple linear regression were used as appropriate. $\mathrm{P}<0.05$ was considered to be significant. 


\section{Results}

Three hundred patients met the inclusion criteria of which 86 (28.7\%) were males and 214 (71.3\%) were females. The mean age of the patients was $37.7 \pm 9.6$ years. However, the mean age was significantly higher in males than in females $(42.4 \pm 10.8$ Vs $35.8 \pm 8.4, \mathrm{P}<0.05)$.

Table 1: Clinical Characteristics Of The Study Population

\begin{tabular}{|l|l|l|}
\hline Parameters & Mean \pm SD, $(\%)$ & P - value \\
\hline Age & $37.7 \pm 9.6$ & \\
Male & $42.4 \pm 10.8$ & \\
Female & $35.8 \pm 8.4$ & $<0.0001$ \\
\hline Sex & & \\
Male & $86(28.7)$ & \\
Female & $214(71.3)$ & \\
\hline
\end{tabular}

Table 2 showed that majority of the patients were females (79.0\% Vs $21.0 \%)$, married (64.8\% Vs $49.3 \%)$ and were in the $31-40$ age category ( $40.8 \%$ Vs $49.3 \%$ ) in both groups. Moreso, the mean age and BMI were significantly higher in HIV patients with $\mathrm{CD}_{4}$ cells greater than $200 / \mu \mathrm{l}$ than those with $\mathrm{CD}_{4}$ cells less than $200 / \mu 1(38.3 \pm 10.2$ Vs $35.8 \pm 7.1,24.4 \pm 4.7$ Vs $21.4 \pm 3.5, \mathrm{P}<0.05)$.

Table 2: Socio - Demographic And Clinical Characteristics Of Patients

\begin{tabular}{|l|l|l|l|}
\hline Variable & $\begin{array}{l}\text { HIV- patients with } \mathrm{CD}_{4} \text { cells }>200 / \mu \mathrm{l} \\
\mathrm{n}=233) \\
\text { Mean } \pm \mathrm{SD}, \%\end{array}$ & $\begin{array}{l}\text { HIV- patients with } \mathrm{CD}_{4} \text { cells }<200 / \mu 1 \\
(\mathrm{n}=67) \\
\text { Mean } \pm \mathrm{SD}, \%\end{array}$ & $\mathrm{P}-$ value \\
\hline Age & $38.3 \pm 10.2$ & $35.8 \pm 7.1$ & 0.007 \\
\hline Age category & $2(0.9)$ & $1(1.5)$ & \\
$<20$ years & $52(22.3)$ & $18(26.9)$ & \\
$21-30$ years & $95(40.8)$ & $33(49.3)$ & 0.059 \\
$31-40$ years & $56(24.0)$ & $14(20.9)$ & \\
$41-50$ years & $22(9.4)$ & $1(1.5)$ & \\
$51-60$ years & $6(2.6)$ & $0(0.0)$ & \\
$>$ 60 years & & $22(25.6)$ & \\
\hline Sex & $64(74.4)$ & $45(21.0)$ & \\
Male & $169(79.0)$ & $25(37.3)$ & \\
Female & & $33(49.3)$ & \\
\hline Marital status & $56(69.1)$ & $0(0.0)$ & \\
Single & $151(64.8)$ & $9(13.4)$ & \\
Married & $4(1.7)$ & $21.4 \pm 3.5$ & 0.044 \\
Divorced & $22(9.4)$ & $0.88 \pm 0.03$ & 0.331 \\
Widows & $24.4 \pm 4.7$ & & \\
\hline Body mass index (BMI) & $0.89 \pm 0.03$ & & \\
\hline Waist-hip-ratio & & & \\
\hline
\end{tabular}

In table 3, the mean HDL is significantly higher in HIV patients with $\mathrm{CD}_{4}$ cells greater than $200 / \mu 1$ while the TG and AIP are significantly higher in HIV patients with $\mathrm{CD}_{4}$ cells less than $200 / \mu \mathrm{l}(\mathrm{P}<0.05)$. Also, HIV patients with $\mathrm{CD}_{4}$ cells less than $200 / \mu 1$ were more in the AIP high risk group as compared those with $\mathrm{CD}_{4}$ cells greater than $200 / \mu \mathrm{l}$ but this was not statistically significant ( $61.2 \% \mathrm{Vs} 46.4 \%, \mathrm{P}>0.05)$.

Table 3: Lipid Profile Pattern Based On $\mathrm{CD}_{4}$ Count Levels

\begin{tabular}{|l|l|l|l|}
\hline Variable & $\begin{array}{l}\text { HIV- patients with } \mathrm{CD}_{4} \text { cells }>200 / \mu \mathrm{l} \\
(\mathrm{n}=233) \\
\text { Mean } \pm \mathrm{SD}, \%\end{array}$ & $\begin{array}{l}\text { HIV-patients with } \mathrm{CD}_{4} \text { cells }<200 / \mu \mathrm{l} \\
(\mathrm{n}=67) \\
\text { Mean } \pm \mathrm{SD}, \%\end{array}$ & $\mathrm{P}$ - value \\
\hline TC & $3.8 \pm 0.43$ & $3.7 \pm 0.56$ & 0.196 \\
\hline HDL & $0.9 \pm 0.26$ & $0.7 \pm 0.22$ & $<0.0001$ \\
\hline LDL & $2.21 \pm 0.37$ & $2.15 \pm 0.51$ & 0.424 \\
\hline TG & $1.57 \pm 0.73$ & $1.89 \pm 0.86$ & 0.006 \\
\hline AIP & $0.19 \pm 0.28$ & $0.36 \pm 0.30$ & $<0.0001$ \\
\hline AIP risk & $101(43.3)$ & $20(29.9)$ & \\
Low risk & $24(10.3)$ & $6(9.0)$ & 0.09 \\
Medium risk & $108(46.4)$ & $41(61.2)$ & \\
High risk & & & \\
\hline
\end{tabular}

Majority of the patients 149 (49.7\%) and more females were in the high risk category. The mean HDL and LDL were significantly higher in the low risk category as compared to the medium and high risk categories $(1.1 \pm 0.23$ Vs $0.9 \pm 0.17$ Vs $0.16,2.3 \pm 0.33$ Vs $2.2 \pm 0.27$ Vs $2.1 \pm 0.48$ respectively, $\mathrm{P}<0.05)$. However, the mean TC and TG were significantly higher in the high risk category as compared to the low and medium risk 
categories $(\mathrm{P}<0.05)$. The age is higher while the $\mathrm{CD}_{4}$ count levels are lower in the high risk category compared with the other categories but not statistically significant $(\mathrm{P}>0.05)$.

Table 4: Clinical And Biochemical Characteristics Of Patients According To AIP Risk

\begin{tabular}{|l|l|l|l|l|}
\hline Variable & $\begin{array}{l}\text { Low risk } \\
(\mathrm{AIP}<0.1) \\
(\mathrm{n}=121)\end{array}$ & $\begin{array}{l}\text { Medium risk } \\
(\text { AIP 0.1 }-0.24) \\
(\mathrm{n}=30)\end{array}$ & $\begin{array}{l}\text { High risk } \\
(\mathrm{AIP}>0.244) \\
(\mathrm{n}=149)\end{array}$ & P - value \\
\hline Age & $36.4 \pm 9.4$ & $35.9 \pm 8.1$ & $39.1 \pm 9.9$ & 0.267 \\
\hline $\begin{array}{l}\text { Sex } \\
\text { Male }\end{array}$ & $\begin{array}{l}27(22.3) \\
\text { Female }\end{array}$ & $\begin{array}{l}8(26.7) \\
22(73.3)\end{array}$ & $\begin{array}{l}51(34.2) \\
98(65.8)\end{array}$ & 0.095 \\
\hline BMI & $24.0 \pm 4.8$ & $24.5 \pm 5.0$ & $23.4 \pm 4.3$ & 0.152 \\
\hline WHR & $0.89 \pm 0.03$ & $0.89 \pm 0.03$ & $0.89 \pm 0.03$ & 0.247 \\
\hline CD ${ }_{4}$ count & $423.9 \pm 225.4$ & $428.2 \pm 269.5$ & $356.0 \pm 256.5$ & 0.493 \\
\hline TC & $3.8 \pm 0.42$ & $3.7 \pm 0.37$ & $3.9 \pm 0.5$ & 0.043 \\
\hline HDL & $1.1 \pm 0.23$ & $0.9 \pm 0.17$ & $0.7 \pm 0.16$ & 0.003 \\
\hline LDL & $2.3 \pm 0.33$ & $2.2 \pm 0.27$ & $2.1 \pm 0.48$ & $<0.0001$ \\
\hline TG & $1.0 \pm 0.26$ & $1.3 \pm 0.25$ & $2.3 \pm 0.6$ & $<0.0001$ \\
\hline
\end{tabular}

In the bivariate correlation analysis of $\mathrm{CD}_{4}$ count levels with lipid profile, $\mathrm{HDL}$ is strongly positively correlated while TG and AIP are negatively correlated.

Table 5: Correlation Of $\mathrm{CD}_{4}$ With Lipid Parameters

\begin{tabular}{|l|l|l|}
\hline Variable & Correlation ( R value) & $\mathrm{P}$ - value \\
\hline TC & 0.092 & 0.111 \\
\hline HDL & $0.284^{* *}$ & $<0.0001$ \\
\hline LDL & 0.051 & 0.380 \\
\hline TG & $-0.148^{*}$ & 0.010 \\
\hline AIP & $-0.222^{* *}$ & $<0.0001$ \\
\hline
\end{tabular}

\section{Discussion}

This study assessed the lipid profile and AIP in HAART - naïve HIV patients with a view of determining the relationship of these parameters with $\mathrm{CD}_{4}$ count levels. Factors that may be associated with atherogenesis and cardiovascular disease risk in HIV infected patients include dyslipidemia either from the effect of HIV on lipid metabolism or worsen by the use of ART as lipid and AIP abnormalities have been observed in both HIV-infected patients on ARV therapy and ARV-naïve patients [13,17,18].

The study had more female participants but the mean age was significantly higher in males. Majority of the patients were in the younger age categories in both groups while the mean age and BMI were significantly lower in patients with more severity of HIV based on the $\mathrm{CD}_{4}$ count levels.

The female preponderance in our study is similar to findings in studies on HIV patients in our environment $[13,19]$. Also, the findings of younger patients in this study are similar to that from other studies $[13,18]$. The mean age and BMI values in this study were similar to the values obtained in some previous studies on HAART- naïve HIV patients in Nigeria [13, 17, 18, 19].

Dyslipidaemia plays a prominent role as a risk factor for CVD, mainly characterized by increased TG, decreased HDL-c and accumulation of small dense LDL-c particles when the level of LDL-c are often normal. The mean values of TC, HDL-c and LDL-c in this study were low and these values were even lower in HIV patients with more severity of the disease based on the $\mathrm{CD}_{4}$ count levels. These findings are similar to reports from previous study on HAART - naïve HIV patients in Eastern and Northern Nigeria [13, 19].

However, in our study the mean HDL-c value was significantly lower in HIV patients with $\mathrm{CD}_{4}$ cell count $<200 / \mu 1$. Also, the TG levels were significantly higher in HIV patients with more severity of the disease and depressed immunity and this is similar to previous reports from other studies [18, 20].

Moreso, it has been shown that increased TG tends to occur with profound immunosuppression [7]. It is postulated that in HIV, high levels of TG occur due to the presence of inflammation, resulting in elevation of interferon- $\alpha$ which interferes with TG clearance [21] .

A number of lipid related parameters have been used to predict the risk of coronary artery disease. According to Grover et al either the ratio of LDL-c/HDL-c or TC/HDL-c is the best related predictor of future cardiovascular events [22]. However, the log of TG/HDL-c was later shown to be a more accurate predictor of heart diseases, closely correlated with the LDL-c particle size and could serve as an indicator of the atherogenic lipoprotein phenotype [23].

The value of AIP indicates a balance between the actual concentration of plasma TG and HDL-c, which predetermine the direction of the cholesterol transport in the intravascular pool toward atherogenic LDL-c 
or beneficial HDL-c [24]. Clinical studies have shown that AIP predicts cardiovascular risk and that it is an easily available risk marker and a useful measure of response to treatment [25].

In this study, the value of AIP is higher in HIV patients with lower $\mathrm{CD}_{4}$ cell counts, while patients with high risk AIP had significantly low levels of HDL-c, LDL-c and high levels of TG. Also, lower $\mathrm{CD}_{4}$ count is associated with higher atherogenic risk profile as shown by the high risk AIP. The findings in our study are similar to that in a previous study on HAART - naïve HIV patients in Eastern Nigeria [13].

Our study also showed that $\mathrm{CD}_{4}$ count was closely related to atherogenic risk profile and index. In that, those who had lower $\mathrm{CD}_{4}$ count (hence lower immunity) had a higher atherogenic risk profile. Hence, low HDL, high TG and AIP were predictive of the $\mathrm{CD}_{4}$ count and this atherogenic profile can be used to identify HIV patients with high cardiovascular risk. There is a similar report from a study in Nigeria by Cajetan et al [13].

The AIP is a simple tool which can easily be used to assess atherogenic risk in patients who may have apparently normal lipid profile using other parameters.

This study is not without limitations, it was a cross - sectional single centre study and the participants were not followed up after the commencement of HAART.

Despite these limitations, our findings indicate that HAART - naïve HIV patients have variations in their lipid profiles based on the $\mathrm{CD}_{4}$ count levels.

\section{Conclusion}

Dyslipidemia is common in HAART-naive HIV patients in our environment. The AIP is more useful than single lipid parameters such as LDL-c or TC in identifying patients with atherogenic risk. In addition, reduced immunity is associated with increased atherogenicity. It is recommended that lipid assessment using lipid ratios such as the AIP should be carried out prior to initiation of therapy in patients with HIV infection.

\section{References}

[1]. UNAIADS, UNAIDS report on global HIV/AIDS epidemic: HIV infection rates decreasing in several countries but global number of people living with HIV continues to rise, Geneva 2005.

[2]. $\quad$ A.S.Suu Kyi, and M.Sidibe, UNAIDS World AIDS Day Report, 2012.

[3]. UNAIDS, HIV and AIDS in Nigeria, UNAIDS Gap Report, 2016.

[4]. UNAIDS, Overview of the global AIDS Epidemic, 2006 Report on the global AIDS epidemic, UNAIDS, 2006

[5]. C.M.Wyatt, R.R.Arons, and P.E.Klotman,Acute renal failure in hospitalized patients with HIV: risk factors and impact on inhospital mortality, AIDS,20(4), 2006,561-565.

[6]. K.V.Heath,R.S. Hogg, K.J.Chan, M.Harris, V.Montessori, M.V.O’Shaughnessy, and J.S.Montanera , Lipodystrophy-associated morphological,cholesterol and triglyceride abnormalities in a population-based HIV/AIDS treatment database, AIDS, 15(2),2001,231-239.

[7]. W.M.El-Sadr, C.M.Mullin,A. Carr,C. Gibert, C.Rappoport, F.Visnegarwala, C.Grunfeld , and S.S.Raghavan ,Effects of HIV disease on lipid, glucose and insulin levels: Results from a large antiretroviral-naive cohort, HIV Medicine,6(2),2005,114-121.

[8]. H.H.Schmidt, G.Behrens, J.Genschel, M.Stoll, A.Dejam, R.Haas, P.Manns, and R.E.Schmidt, Lipid evaluation in HIV-1-positive patients treated with protease inhibitors, Antiretroviral Therapy,4(3),1999,163-170.

[9]. B.F.Asztalos, E.J.Schaefer, K.V.Horvath, C.E.Cox, S.Skinner, J.Gerrior , S.L.Gorbach , and C.Wanke, Protease inhibitor-based HAART, HDL, and CHD risk in HIV infected patients, Atherosclerosis, 184(1), 2006, 72-77.

[10]. H.Rose, I.Woolley, J.Hoy, A.Dart, B.Bryant, A.Mijch, A.Mijch , and D.Sviridov, HIV infection and high-density lipoprotein: The effect of the disease vs the effect of treatment, Metabolism ,55(1),2006,90-95.

[11]. H.Rose, J.Hoy, I.Woolley, U.Tchoua, M.Bukrinsky, A.Dart,and D.Sviridov, HIV infection and high density lipoprotein metabolism, Atherosclerosis, 199(1),2008,79-86.

[12]. P.L.Luz, D.Favarato, J.R.F.Junior, P.Lemos, and A.C.P.Chagas, High ratio of triglycerides to HDL-cholesterol predicts extensive coronary disease, Clinics, 63(4), 2008, 427-32.

[13]. C.O.Cajetan, E.Y.Ekenechukwu, O.I.Michael, J.C.Chinwe, and I.N.Uchenna ,Atherogenic index of plasma in highly active antiretroviral therapy-naive patients with human immunodeficiency virus infection in Southeast Nigeria, Indian Journal of Endocrinology and Metabolism, 18(5), $2014,631-636$.

[14]. Report of WHO Scientific Group, Cardiovascular Disease Risk Factors; New Areas for Research, WHO Technical Report Series, No. 841, Geneva: WHO, 1994.

[15]. M.Dobiásová, AIP - atherogenic index of plasma as a significant predictor of cardiovascular risk: From research to practice, Vnitr Lek, 52(1), 2006, 64-71.

[16]. W.T.Friedwald, R.I.Levy, and D.S.Fredrickson,Estimation of the concentration of low density lipoprotein cholesterol without use of preparative ultracentrifuge, Clinical Chemistry, 18(6),1972,499-504

[17]. R.Yusuf, A.I.Sambo, M.H.Mohammed, and H.Abdulaziz, Lipid profile of HIV/AIDS patients attending antiretroviral clinic in Zaria, North - Western Nigeria, Sub-saharan African Journal of Medicine, 1(1),2014,31-35.

[18]. O.OAdewole, S.Eze, Ye.Betiku, E.Anteyi, I.Wada, Z.Ajuwon, and G.Erhabor , Lipid profile in HIV/AIDS patients in Nigeria, African Health sciences, 10(2),2010,144-149.

[19]. C.Daniyam, and M.Iroezindu, Lipid profile of anti-retroviral treatment naive HIV-infected patients in Jos, Nigeria, Annals of Medical Health Sciences and Research, 3(1), 2013, 26-30.

[20]. T.S.Iffen, H.Efobi, C.A.Usoro,and N.E. Udonwa, Lipid profile of HIV-positive patients attending University of Calabar Teaching Hospital Calabar, Nigeria, World Journal of Medical Sciences,5(9),2010,89-93. 
[21]. S.B. Haugaard, O.Andersen, S.B.Pedersen, F.Dela, M.Fenger, B.Richelsen, S.Madsbad, J.Iversen, Tumor necrosis factor alpha is associated with insulin-mediated suppression of free fatty acids and net lipid oxidation in HIV-infected patients with lipodystrophy, Metabolism,55(2),2006,175-82.

[22]. S.A.Grover, C.Levinton, and S.Panquet, Identifying adults at low risk for significant hyperlipidemia: a validated clinical index, Journal of Clinical Epidemiology, 52(1), 1999, 49-55.

[23]. M.Dobiasova, and J.Frohlich, "The plasma parameter log (TG/HDL-C-C) as an atherogenic index", Clinical Biochemistry, 34(7), 2001, 583-588.

[24]. M.Dobiasova, and J.Frohlich, "Understanding the mechanism of LCAT reaction may help to explain the high predictive value of LDL-C-HDL-C cholesterol ratio", Physiology Research, 47(6), 1998, 387-397.

[25]. J.Frohlich, and M.Dobiasova, "Fractional esterification rate of cholesterol and ratio of triglyceride to HDL-C are powerful predictors of positive findings on coronary angiography", Clinical Chemistry, 49(11), 2003, 1873-1880. 